\title{
AVANZANDO EN LA MEDICIÓN DE LAS REDES SOCIALES DIGITALES: UNA PROPUESTA PARA SU ANÁLISIS DESDE UNA PERSPECTIVA INSTITUCIONALISTA
}

\author{
Francisco Rojas-Martín \\ Universidad Autónoma de Madrid, España \\ francisco.rojas@inv.uam.es \\ J. Ignacio Criado \\ Universidad Autónoma de Madrid, España \\ Ignacio.criado@uam.es
}

\section{RESUMEN}

La difusión entre las administraciones públicas de las redes sociales digitales como herramientas para potenciar su relación con la sociedad se ha producido de manera imparable en la última década y media. Este artículo, a partir del enfoque del Enacting Electronic Government Success (EEGS), propone un modelo de análisis que sirva para enmarcar futuras investigaciones en el ámbito de las redes sociales en la administración pública. Concretamente, a través de una adaptación del EEGS a las redes sociales, se tratará de analizar los resultados de las interacciones entre las administraciones públicas y las tecnologías de la información y la comunicación, a partir del estudio de los factores institucionales, organizativos y medioambientales.

Palabras clave: Redes sociales digitales, Gobierno Electrónico, Neoinstitucionalismo, Administración pública. 


\title{
ADVANCING IN THE DIGITAL SOCIAL NETWORKS MEASUREMENT: A PROPOSAL FOR ITS ANALYSIS FROM AN INSTITUTINALIST PERSPECTIVE
}

\begin{abstract}
Digital social networks dissemination among public administrations as a tool to promote their relation with the society has been unstoppably produced in the last fifteen years. This article, from the Enacting Electronic Government Success (EEGS) approach, proposes an analysis model that serves as a framework for future research in the field of social networks in public administration. Specifically, through an adaptation of EEGS in social networks, the results of the interaction between public administrations and information and communication technology will be attempted to analyze from the study of institutional, organizational and environmental factors.
\end{abstract}

Keywords: Digital social networks, Electronic government, Neoinstitutionalism, Public administration. 


\section{INTRODUCCIÓN}

La difusión de las redes sociales digitales (RSD) se ha producido de una manera acelerada, especialmente en la última década y media. Redes sociales que crecían vertiginosamente y que eran objeto de estudio como SixDegrees han desaparecido o van camino de hacerlo, mientras que otras que han surgido más recientemente, como Facebook o Twitter, cuentan con centenares de millones de usuarios en todo el mundo (Boyd y Ellison 2007). En efecto, los datos muestran un elevado nivel de penetración en las sociedades avanzadas de estas tecnologías sociales. Se calcula que existen en torno a los 3.000.000.000 de usuarios en todo el mundo, lo que nos ayuda a comprender la dimensión que está alcanzando este fenómeno.

Existe un consenso en la literatura sobre la rápida difusión de las RSD entre las administraciones públicas en todos sus niveles (Agostino 2013, Bonson, Royo y Ratkai 2013, Criado y Rojas-Martín 2015, De Saulles 2011, Graham y Avery 2013, Mainka, et. al 2014), Mickoleit 2014, Maultasch Oliveira y Welch 2013, Mossberger, Wu y Crawford 2013, Panagiotopoulos 2012), si bien todavía queda mucho trabajo por delante para comprender cuáles son las dinámicas que se están llevando a cabo en el proceso de adopción de estas tecnologías. En este sentido, interesa conocer los factores que permiten lograr el éxito de las administraciones públicas en el uso de estas tecnologías principalmente en términos de interacción y valoración desde una perspectiva ciudadana. Para ello, como veremos más adelante, se propone encontrar las relaciones causales entre diversos tipos de factores identificados como relevantes en el proceso con respecto al grado de influencia ejercido sobre los seguidores de las cuentas en RSD de las administraciones públicas.

En este contexto de creciente interés por el uso de las redes sociales digitales en el sector público, es necesario desarrollar marcos analíticos específicos que permitan encuadrar adecuadamente el estudio de esta incipiente disciplina. Por ello, este artículo tiene como objetivo realizar una propuesta de modelo conceptual para el análisis de las redes sociales y la administración pública. Concretamente, la pregunta de investigación que se pretende responder va encaminada a conocer cuáles son los factores para el éxito de las redes sociales en el sector público. Para ello, se va a partir del modelo Enacting Electronic Government Success (EEGS) propuesto por Gil-García (2012). Este modelo teórico neoinstitucionalista, ha sido aplicado previamente al estudio del gobierno electrónico, y según su autor, se inspira en los trabajos de Jane Fountain (2001), en los que se estudiaban las interacciones generadas entre el gobierno y las tecnologías de la información en Estados Unidos. 
Este artículo se encuadra en una línea de investigación sobre las redes sociales digitales en la administración pública española y tratará de cubrir un espacio que necesita desarrollar a nivel teórico y empírico. La propuesta que aquí se presenta se viene desarrollando desde el año 2013, nutriéndose a lo largo de este tiempo de las contribuciones realizadas en diversos seminarios y congresos académicos. Este estudio propone un modelo de análisis que sirva para enmarcar futuras investigaciones en el ámbito de las redes sociales en la administración pública. Concretamente, a través de una adaptación del EEGS a las redes sociales, se tratará de analizar los resultados de las interacciones entre las administraciones públicas y las tecnologías de la información y la comunicación, mediante el estudio de los factores institucionales, organizativos y medioambientales.

La siguiente sección de este estudio presenta la revisión bibliográfica, la sección segunda estará dedicada al enfoque neoinstitucionalista para el estudio de la tecnología en la administración pública, en la sección tercera se presenta la propuesta para la adaptación del modelo EEGS para el estudio de las redes sociales digitales. Finalmente se ofrecen algunas conclusiones preliminares sobre el modelo de análisis y los desafíos que deben superarse para alcanzar los objetivos propuestos en esta investigación durante la segunda fase del estudio.

\section{REVISIÓN BIBLIOGRÁFICA: ¿¿REDES SOCIALES DIGITALES PARA MEJORAR LA RELACIÓN CON LA CIUDADANÍA?}

La rápida difusión de las redes sociales digitales en las administraciones públicas de todo el mundo ha generado un amplio interés desde el ámbito académico. El estudio de este fenómeno es relativamente reciente y tiene un carácter multidisciplinario (Boyd y Ellison 2007). Incluye las áreas de la informática, los negocios, el Derecho, la Sociología, la Ciencia Política y la Gestión Pública, entre otros. Todas estas disciplinas tienen un objetivo común, comprender unas tecnologías cuyo componente social las convierten en potencialmente disruptivas. Así lo consideran algunos autores, como Mergel (2012b), que sugiere que la aplicación de las redes sociales en el sector público puede ser considerada como la quinta oleada de tecnologías de la información y la comunicación (TIC). Un estadio de desarrollo, que en el caso de las administraciones públicas, nos situaría en un proceso de transición desde el gobierno electrónico (e-gobierno) al Gobierno 2.0, caracterizado por la co-creación de los servicios y por la toma de decisiones públicas de manera más inclusiva (Chun et al. 2010).

En esta fase de desarrollo aún incipiente, la literatura se refiere y define las redes sociales digitales de manera muy heterogénea. En este artículo vamos a utilizar los conceptos de redes sociales digitales, redes sociales y tecnologías 
sociales de manera análoga. En cuanto a su definición, proponemos una definición integradora: las RSD son tecnologías sociales derivadas de la web 2.0 que permiten la generación de comunidades virtuales a partir de la conexión, generación, interacción e intercambio de información de un colectivo ilimitado de personas que comparten unos intereses comunes (Criado y Rojas-Martín 2013a, Kaplan y Haenlein 2010, Chun et al. 2010, Boyd y Ellison 2007). En síntesis, estamos hablando de un tipo de tecnología que sustenta su valor principalmente en su dimensión social.

El uso de las redes sociales digitales entre las administraciones públicas y la ciudadanía ofrece nuevas oportunidades para comprender la esfera pública. Las interacciones ofrecen datos que merecen ser utilizados para comprender mejor a la ciudadanía: "lo que hace que las redes sociales sean únicas, no es que permitan conectar con extraños, sino más bien que habilitan a los usuarios a articular y hacer visible sus redes sociales" (Boyd y Ellison 2007: 211). En este sentido, la información extraída de las redes sociales permite comprender mejor la forma en que opera la esfera pública, crear nuevas formas de gobernar, y/o mejorar las diferentes formas de participación (Bailey y Singleton 2010, Bertot et al. 2012a, Bonsón et al 2012, Criado et al 2011, Criado y Rojas-Martín 2012). Además, ofrecen la oportunidad de conocer los vínculos sociales que se generan entre los ciudadanos, las administraciones públicas y otros actores sociales de una manera desconocida hasta el momento.

Las redes sociales representan un paso adelante en la evolución de la innovación tecnológica en el sector público. Por ello, su introducción podría favorecer la comunicación entre los ciudadanos y las instituciones, ya que permiten a éstas últimas poder interactuar por primera vez con los ciudadanos de una manera bidireccional (Mergel 2012a). Suponen un paso adelante respecto a los medios de comunicación tradicionales, ya que las redes sociales "se basan en el contenido generado por el usuario" (Bertot et al. 2012a). Por lo tanto, las principales redes sociales, que cuentan en la actualidad con millones de usuarios, no existirían sin el contenido generado por sus usuarios (De Saulles 2011). En suma, el valor ańadido de las RSD radica en que los contenidos se desarrollan por la ciudadanía de una manera más horizontal y abierta, lo que sumado a la bidireccionalidad de estas herramientas, puede facilitar la interacción con las instituciones públicas.

Desde la perspectiva de los ciudadanos, las redes sociales ofrecen una plataforma más adecuada para la participación directa en la toma de decisiones. En efecto, permiten la transmisión de la opinión de los ciudadanos de una manera más simple, más rápida y más directa, fomentando la creación de unas instituciones públicas mejor informadas, 
más innovadoras, más centradas en el ciudadano y, por lo tanto, más democráticas (Bonsón et al. 2012, Chun et al. 2010). Dentro del sector público, estas tecnologías también podrían "permitir la colaboración distribuida y la creación de inteligencia colectiva a gran escala, a todos los niveles, desde el local al federal" (Chun et al. 2010: 5). Por lo tanto, las redes sociales pueden mejorar la transparencia administrativa, ya que los ciudadanos colaboran en el proceso de establecimiento de la agenda (Meijer y Thaens 2013). Durante los últimos años, diferentes organismos públicos e instituciones privadas de todo el mundo han hecho algunos avances en este campo.

Desde la perspectiva de la provisión de los servicios públicos, las redes sociales pueden facilitar la incorporación de los ciudadanos en el proceso. Basadas en la filosofía de la Web 2.0, estas tecnologías sociales no sólo implican elementos interactivos, sino que también incorporan actitudes de colaboración en línea con el enfoque de muchos-a-muchos (Bertot et al 2010). Por lo tanto, favorecen la coproducción de los servicios públicos con los ciudadanos, no sólo porque facilitan la posibilidad de realizar opiniones o sugerencias, sino porque además, mejoran la colaboración de los ciudadanos en la elaboración de nuevos contenidos y servicios que requieren su proactividad (Nam 2012). Estas potencialidades pueden verse beneficiadas por el hecho de que el incremento de la participación ciudadana en las políticas públicas se está convirtiendo en un importante elemento para la reputación, la autoridad y el impacto de la acción de los organismos públicos (Heidinger et al. 2010). Y por lo tanto, si por un lado existe una demanda ciudadana, y por el otro, las administraciones públicas disponen de la tecnología adecuada para dar respuesta a esa demanda, podemos comprender que la perspectiva de difusión de las redes sociales en el sector público será cada vez más importante.

Por otro lado, existen diversos autores con una visión más crítica y pesimista sobres los efectos reales de las tecnologías en las administraciones públicas. Primeramente, existen dudas sobre que las RSD estén logrando provocar cambios significativos en la línea de las promesas anticipadas (Criado y Rojas-Martín 2013b). En segundo lugar, tenemos aquellos autores que creen que su utilización como herramientas para la apertura excesiva de la información pública puede ofrecer sesgos o efectos nocivos. Según ellos, esta circunstancia puede provocar un alejamiento de los ciudadanos de las instituciones y la generación de una cultura política cínica (Grimmelikhuijsen 2012). También puede provocar una falta de control y una manipulación de los contenidos que derivaría en comportamientos destructivos (Osimo 2008). Adicionalmente, la participación generada mediante estas tecnologías puede verse sesgada debido a los bajos niveles 
de participación, que pueden derivar en una participación restringida a una élite, y por lo tanto de baja calidad (Lin, Bagrow y Lazer 2011, Osimo 2008).

En otros casos se puede estar produciendo un desajuste normativo por la difusión urgente y sin planificación de estas tecnologías. La legislación existente es previa a la irrupción de las redes sociales, lo que provoca disfuncionalidades a la hora de desarrollar las potencialidades de estas herramientas (Mergel 2012a). También existen otros autores que previenen de poner en marcha proyectos tecnológicos sin constatar su necesidad, especialmente ante el importante ratio de fracasos identificados en los proyectos tecnológicos en el sector público, que además vienen acompañados de enormes pérdidas económicas: (Lips 2012, Gil-García 2012). Finalmente, Bannister y Connolly (2012: 221) se plantean si no convendría obtener mayor provecho de las tecnologías ya desarrolladas, y puestas en marcha pero no explotadas convenientemente, antes que lanzarse de nuevo a la carrera de la innovación tecnológica sin tener evidencias sólidas para ello.

\section{EL ENFOQUE NEOINSTITUCIONALISTA PARA EL ANÁLISIS DE LAS TECNOLOGÍAS EN LA ADMINISTRACIÓN PÚBLICA}

Con el objetivo de realizar una propuesta de marco analítico para el estudio de las redes sociales digitales en las administraciones públicas vamos a utilizar el enfoque planteado por el modelo neoinstitucionalista EEGS, elaborado por Gil-García (2012). El neoinstitucionalismo supone un marco teórico idóneo para comprender la influencia de los factores que rodean a la tecnología en los procesos de adopción en las instituciones públicas (Fountain 2001, Orlikowski y Iacono 2001, Criado 2009, LunaReyes y Gil-Garcia 2011, Gil-Garcia 2012). El EEGS está orientado al estudio del gobierno electrónico y en este trabajo se realiza una labor de adaptación para poder emplearlo en el estudio de las RSD.

El EEGS considera que los efectos de las tecnologías de la información y comunicación (TICs) en el sector público, incluidas las redes sociales, no dependen exclusivamente de sí mismas, sino que también dependen de los arreglos institucionales, de las prácticas organizativas y del entorno en el que se desenvuelven. Por lo tanto, si se quieren comprender los resultados de la implantación del gobierno electrónico en las administraciones públicas, se deben estudiar los factores institucionales, organizativos y ambientales que influyen sobre estas tecnologías (Gil-García 2012, Gascó et al. 2012).

Este modelo analítico se inspira en el trabajo previo denominado Technology Enacment Framework. Este marco de análisis fue creado por 
Fountain (2001), a través del cual trató de comprender la influencia de los arreglos institucionales y las formas organizativas en la selección, el diseño, la implementación y el uso de las tecnologías de la información en la administración pública de los Estados Unidos. En su trabajo, Fountain explicaba cómo las tecnologías de la información objetivas se ven influidas por estos factores produciendo enacted technologies (Fountain 2001), que podríamos traducir como tecnologías representadas. Según la autora esta influencia transformaría la tecnología objetiva original, que se vería moldeada por factores institucionales, dando lugar a la tecnología representada. Por ello, afirma "no es sorprendente, que organizaciones similares puedan utilizar los mismos sistemas de información de una manera tan marcadamente diferente" (Fountain 2001: 89). Al mismo tiempo, y de manera inversa, concluía que las tecnologías adoptadas pueden modificar las disposiciones institucionales y organizativas.

Por su parte, Gil-García (2012) se inspira en el Technology Enacment Framework para realizar una propuesta analítica más amplia sobre el gobierno electrónico en las organizaciones públicas. El EEGS es un enfoque integrador que ha sido probado para estudiar las tecnologías de la información en el ámbito de las administraciones públicas. Pero también es un modelo que "podría ser utilizado para comprender cualquier tipo de iniciativa de gobierno electrónico", incluyendo el uso de redes sociales digitales (Gil-García 2012). El modelo EEGS intenta completar el Technology Enacment Framework, incorporando el constructivismo social y otros enfoques integradores. Como resultado, este marco no sólo considera los arreglos institucionales y organizacionales aportados por Fountain (2001), sino que también analiza las condiciones contextuales o ambientales (ver Gráfico 1).

En el modelo EEGS encontramos en primer lugar los factores institucionales. Estos factores son esenciales para comprender adecuadamente los resultados de cualquier proyecto TIC en la administración pública (Luna-Reyes y Gil-Garcia 2011). En palabras de Gil-García (2012: 60), los "arreglos institucionales son elementos muy importantes en la comprensión de cómo se selecciona el diseño, la implementación y la utilización de las tecnologías de la información en las organizaciones públicas". Esto incluye las leyes, los reglamentos, el sistema de gobierno, el control político y los comportamientos culturales o socioestructurales aceptados por los miembros de la organización.

El análisis de los factores instituciones adquiere especial importancia en la administración pública por su especificidad y singulares características. En palabras de Criado (2009: 18), "los procesos de adopción y difusión de las nuevas tecnologías en las organizaciones públicas presentan dificultades 
debido a sus entornos organizativos burocratizados e institucionalizados". Esta consideración muestra la relevancia de tener en cuenta esta dimensión para comprender los elementos que pueden explicar el éxito o el fracaso en la adopción de estas tecnologías sociales.

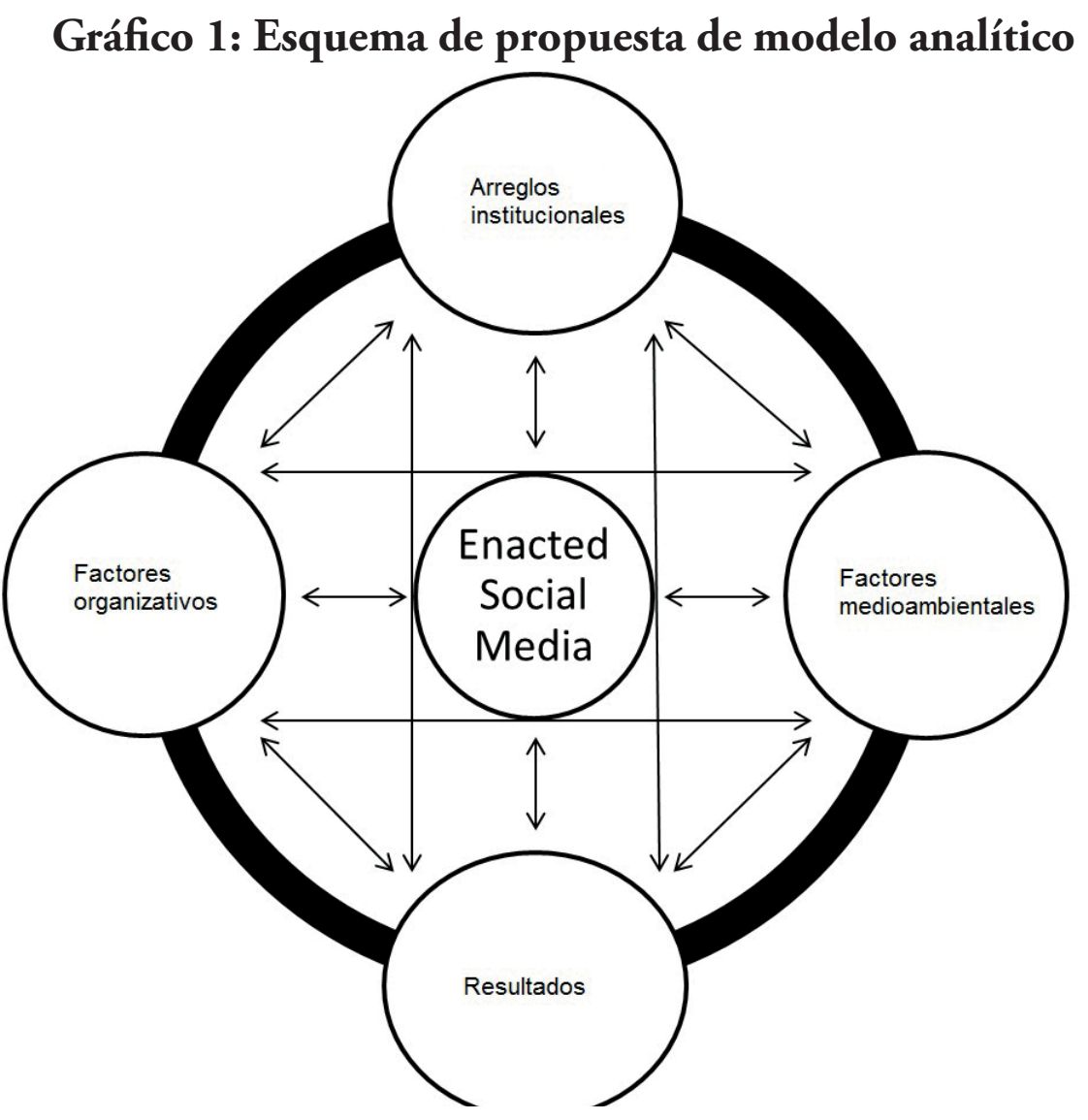

Fuente: Elaboración propia.

En segundo lugar, el modelo EEGS incorpora la dimensión organizativa como factor de análisis. Considera que las estructuras y procesos organizativos, características organizativas y estrategias de gestión tienen una influencia directa como factores de éxito sobre las tecnologías adoptadas (Luna-Reyes y Gil-García 2011). A mbos constructos se integran en el marco EEGS a través de indicadores como el número de personas que trabajan para la organización de tecnologías de la información, la accesibilidad a la formación de los profesionales, el número de canales de comercialización utilizados, el servicio provisto internamente o externamente (Gil-García 2012: 69).

El EEGS incluye una dimensión adicional que se orienta a identificar los factores contextuales o medioambientales que afectan al proceso de adopción de la tecnología en la administración pública. Las condiciones 
ambientales son identificadas por la literatura como factores relevantes "en la configuración de la forma en la que la tecnología de la información está diseñada, implementada y utilizada en las organizaciones" (Gil-García 2012: 61). Sin embargo, la evidencia indica que esta influencia puede estar mediada por variables institucionales o de organización (Gil-García 2012). Por lo tanto, es necesario controlar los efectos provocados por factores ambientales en la tecnología de la información mediante el análisis de los factores organizativos e institucionales. De lo contrario, existe el riesgo de obtener conclusiones sesgadas.

\section{PROPUESTA PARA LA ADAPTACIÓN DEL MODELO EEGS PARA EXPLORAR LOS FACTORES DE ÉXITO EN LA ADOPCIÓN DE LAS TECNOLOGÍAS SOCIALES}

Este artículo va a utilizar el EEGS como punto de partida para elaborar una propuesta de modelo analítico adaptado a las redes sociales digitales. En primer lugar, hemos seleccionado este marco porque previamente ha sido probado con éxito en el sector público. En segundo lugar, se ha tenido en cuenta, ya que como hemos visto, es un modelo utilizado para analizar el gobierno electrónico, pero que también podría ser utilizado para el estudio de las redes sociales en las administraciones públicas (Gil-García 2012). Por último, los enfoques institucionalistas, como el EEGS, son adecuados para comprender fenómenos complejos en las relaciones entre tecnologías de la información y administraciones públicas (Gil-García 2012: 53, Criado 2009: 18).

Como ya hemos señalado, el objetivo de este artículo consiste en adaptar el modelo EEGS para ser utilizado en el estudio de las redes sociales digitales. Este enfoque está orientado al análisis del gobierno electrónico, y en este trabajo, vamos a tratar de adaptarlo a las especifidades de estas tecnologías sociales. Concretamente la pregunta de investigación que se plantea responder mediante la elaboración de esta propuesta analítica es: ¿cuáles son los factores que influyen en el éxito de las redes sociales en el sector público?

Para tratar de responder a esta pregunta de investigación vamos a trabajar con tres variables independientes siguiendo el planteamiento que se realiza en el modelo EEGS. En primer lugar, se van a estudiar las estructuras y procesos organizativos $\left(\mathrm{X}_{1}\right)$. En segundo lugar, se tendrán en cuenta los arreglos institucionales $\left(\mathrm{X}_{2}\right)$ y por último, se incorporarán al análisis las condiciones medioambientales $\left(\mathrm{X}_{3}\right)$. Finalmente, consideraremos como variable dependiente el éxito de las redes sociales digitales en el sector público. Para tratar de alcanzar el objeto de estudio se van a operacionalizar estas tres dimensiones mediante la identificación de indicadores específicos 
al análisis de las redes sociales digitales.

Para operacionalizar el constructo estructuras y procesos organizativos $\left(\mathrm{X}_{1}\right)$, vamos a utilizar los siguientes indicadores: 1) modelo de gestión, relacionado con la forma en la que se ponen en marcha los proyectos tecnológicos (Gascó et al. 2012, Gil-García 2012, Picazo-Vela, GutierrezMartinez y Luna-Reyes 2012, Meijer y Thaens 2013), como por ejemplo la existencia de un plan estratégico de redes sociales, existencia de iniciativas para promover las RSD, evaluación de resultados, tipo de relación contractual del personal de las RSD (temporal o permanente), gestión concentrada o desconcentrada de las redes sociales; 2) tamaño de la organización, medido mediante el número de empleados en la unidad de redes sociales (Gil-García 2012); 3) acceso de los empleados a formación específica (Gil-García 2012), orientado a conocer si existe o no formación específica a los empleados responsables de gestionar las RSD; 4) experiencia de la organización en el uso de las RSD (Gascó et al 2012), en número de años utilizando estas tecnologías por parte de la institución y del personal a su servicio; 5) liderazgo, para conocer si existe un liderazgo claro al frente de la gestión de las RSD (Gascó et al. 2012).

En el caso de los arreglos institucionales $\left(\mathrm{X}_{2}\right)$ serán analizados a través de los siguientes indicadores: 1) compromisos formales sobre transparencia (Luna Reyes 2006, Bonson et al 2012), se va a medir comprobando si existe algún documento específico sobre esta materia; 2) existencia de criterios normalizados para el uso de las redes sociales, como por ejemplo si existen guías de uso que orienten sobre el funcionamiento de las redes sociales dentro de la institución (Gil-Garcia 2012); 3) compromisos formales sobre acceso a la información pública (Luna Reyes 2006, Graham y Avery 2013, Nam 2012, Bertot et al. 2012a); 4) compromisos formales sobre e-administración (Mickoleit 2014, Bertot et al. 2012b); 5) compromisos formales sobre participación ciudadana, se analizará si existe algún tipo de documento específico en este ámbito puesto que puede suponer un incentivo para el uso de tecnologías sociales (Mickoleit 2014, Ellison y Hardey 2014, Linders 2012, Bertot et al. 2012b, Picazo-Vela, GutierrezMartinez y Luna-Reyes 2012); y 6) cultura de la transparencia, orientado a conocer si existen indicaciones formales o informales, o hábitos que indiquen, que se fomenta una cultura de la transparencia (Song y Lee 2013, Meijer y Thaens 2013, Bonson, Royo y Ratkai 2013, Picazo-Vela, Gutierrez-Martinez y Luna-Reyes 2012).

Por último, vamos a analizar las condiciones medioambientales o de entorno $\left(\mathrm{X}_{3}\right)$. Esta dimensión puede afectar a las organizaciones públicas en forma de amenazas u oportunidades (Gascó et al. 2012). En este estudio las condiciones medioambientales están representadas por: 1) desarrollo 
tecnológico, orientado a conocer si existe un contexto adecuado, de modo que "si el acceso a las tecnologías es escaso, sólo unos pocos podrán beneficiarse de su uso" (Gascó 2009:45). Se incluye aquí igualmente el grado de conocimiento de las cuentas institucionales en RSD por parte de la ciudadanía. La esencia de estas tecnologías sociales consiste en crear comunidades en línea y para ello se necesitan personas que sigan a las cuentas oficiales. Esta variable ya ha sido considerada en trabajos previos (Abdelsalam et al. 2013, Agostino 2013, Mickoleit 2014). La recogida de datos se realizará mediante la observación de las cuentas oficiales de las administraciones públicas y se ponderará en base al número total de habitantes; 2) situación económica, se trata de una dimensión muy habitual para medir proyectos tecnológicos puesto que el nivel de la economía influye en la capacidad de las organizaciones (Gil-García 2012, Gascó et al. 2012); 3) demografía, en la que se encuadran los factores como la población, el nivel educativo y la edad (Gil-García 2012); 4) contexto político, incluyendo el partido político del gobierno (Gil-Garcia 2012), la estabilidad política del gobierno, que mide el tiempo que llevan los representantes políticos al frente de las instituciones públicas (Luna Reyes 2006) y el tipo de gobierno, que puede ser de mayoría o coalición (Criado, Martinez-Fuentes, Silván 2012). 


\section{Tabla 1: Operacionalización del modelo analítico}

\begin{tabular}{|c|c|}
\hline Constructo & Indicadores \\
\hline $\begin{array}{c}\text { Estructura y procesos } \\
\text { organizativos/ } \\
\text { características } \\
\text { organizativas y } \\
\text { estrategias de gestión }\end{array}$ & $\begin{array}{l}\text { Existencia de iniciativas para promover las RSD } \\
\text { Existencia de un plan estratégico de RSD } \\
\text { Acceso a formación específica por parte de la } \\
\text { organización } \\
\text { Tamańo de la organización } \\
\text { Experiencia de la organización en el uso de las } \\
\text { RSD } \\
\text { Liderazgo } \\
\text { Evaluación de resultados } \\
\text { Gestión concentrada o desconcentrada de las RSD } \\
\text { Tipo de relación contractual del personal de las } \\
\text { RSD }\end{array}$ \\
\hline Arreglos instutcionales & $\begin{array}{l}\text { Compromisos formales sobre transparencia } \\
\text { Existencia de criterios normalizados para el uso de } \\
\text { las redes sociales } \\
\text { Compromisos formales sobre acceso a información } \\
\text { pública } \\
\text { Compromisos formales sobre e-administración } \\
\text { Compromisos formales sobre participación } \\
\text { Cultura de la transparencia }\end{array}$ \\
\hline $\begin{array}{l}\text { Condiciones } \\
\text { ambientales }\end{array}$ & $\begin{array}{l}\text { Tecnología } \\
\text { Economía } \\
\text { Demogragía } \\
\text { Política }\end{array}$ \\
\hline
\end{tabular}

Fuente: Elaboración propia.

El objeto de estudio de esta propuesta es conocer cuáles son las variables explicativas del éxito del uso de las redes sociales digitales en las administraciones públicas $(\mathrm{Y})$. La propuesta podrá ser aplicada al estudio de cualquier institución pública, en cualquiera de sus niveles o en base a la función que desempeñen. Definir el éxito de las RSD no es un asunto sencillo porque se trata de un término con un cierto nivel de abstracción que puede ser entendido de múltiples maneras. Existe una línea de estudio que previamente ha discutido sobre esta problemática y tratado de medir el éxito en la adopción de la tecnologías en la administración pública (Mergel 2013, Luna-Reyes, Mellouli y Bertot 2013, Hofmann et al. 2013, Gil-García 2012). En unos casos se ha puesto el énfasis en el análisis de contenidos, identificando aquellos que ofrecen mejores resultados en 
términos de interacciones positivas (Hofmann et al. 2013, Magnusson, Bellström y Thoren 2012). En nuestro caso, enfocaremos el estudio desde una perspectiva neoinstitucional, mediante el análisis de los factores explicativos del éxito de las RSD en la administración pública (Gil-Garcia 2012).

La variable dependiente, éxito del uso de las RSD (Y), se va a medir a partir del indicador ofrecido por la escala de influencia Klout Score. El estudio de la influencia en redes sociales a través de esta herramienta ha sido utilizado en estudios previos (Anger y Kittl 2011, Edwards et al. 2013). Esta escala de medición ampliamente extendida nos permite comprender cuáles son las cuentas con mayor número de seguidores, con mayor actividad, que mayor interacción producen y con una valoración más positiva. En suma, un conjunto de variables que han sido utilizadas en investigaciones previas en este campo de estudio para conocer el nivel de éxito en el uso de las redes sociales de las organizaciones (Kaplan y Haenlein 2010: 65, Mickoleit 2014).

El índice Klout Score es el más extendido a nivel mundial contando con más de 620.000.000 de perfiles (Klout 2015). Para determinar el Klout Score de un perfil, la aplicación analiza los contenidos de las redes sociales y se asigna una puntuación entre 1 y 100 a los usuarios, siendo 0 el nivel de influencia mínimo y 100 el nivel de influencia máximo. Para determinar la puntuación la aplicación mide el tamaño de la red del usuario y correlaciona su contenido con el uso que el resto de usuarios de su red hacen de él. No es un índice meramente cuantitativo que mida el volumen de contenidos generados o de seguidores de un perfil, si no la capacidad de influir que tiene al transmitir sus contenidos (Edwards et al. 2013). Lo anterior es esencial porque permite capturar adecuadamente, con potencial comparativo y a través de un índice sintético, la medida de éxito de las organizaciones públicas dentro de las redes sociales (variable dependiente de este estudio).

Presentadas las variables de estudio, y para completar esta propuesta, es el momento de exponer las hipótesis que completan el marco de análisis. Para ello, partiremos de los mismos planteamientos que se desarrolla en el modelo EEGS (Gil-García 2012). Como hemos visto, las condiciones medioambientales pueden influir directamente en el éxito del gobierno electrónico, pero además hay que tener en cuenta las relaciones indirectas a través de las estructuras y procesos organizativos (Gil-García 2012), ahora bien ¿servirá para los proyectos de adopción de RSD? En la misma línea, los factores institucionales influyen directamente en el éxito del gobierno electrónico, pero también hay que tener en cuenta las relaciones indirectas producidas a través de las estructuras y procesos organizativos 
(Gil-García 2012) ¿se confirmará esta relación en las RSD? Por último, los factores organizativos influyen directamente en el éxito del gobierno electrónico (Gil-Garcia 2012), ¿influirán también en el éxito de las RSD de las administraciones públicas?

Las hipótesis de investigación de este trabajo serán:

$\mathrm{H}_{1}$ : Las condiciones medioambientales tienen una relación directa en el éxito de la adopción de RSD en las administraciones públicas.

$\mathrm{H}_{2}$ : Las condiciones medioambientales tienen una relación indirecta en el éxito de la adopción de RSD en las administraciones públicas, a través de los arreglos institucionales.

$\mathrm{H}_{3}$ : Las condiciones medioambientales tienen una relación indirecta en el éxito de la adopción de RSD en las administraciones públicas, a través de las estructuras y procesos organizativos.

$\mathrm{H}_{4}$ : Los arreglos institucionales tienen una relación directa en el éxito de la adopción de RSD en las administraciones públicas.

$\mathrm{H}_{5}$ : Los arreglos institucionales tienen una relación indirecta en el éxito de la adopción de RSD en las administraciones públicas, a través de las estructuras y procesos organizativos.

$\mathrm{H}_{6}$ : Las estructuras y procesos organizativos tienen una relación directa en el éxito de la adopción de RSD en las administraciones públicas.

Se conocerán los resultados de este marco analítico y la validez de sus hipótesis una vez sea aplicado este modelo utilizando datos empíricos, labor a la que invitamos a todas aquellas personas interesadas en desarrollar este esquema. Aquí se sostiene que esta propuesta analítica puede, potencialmente, aplicarse a cualquier entorno administrativo, en la medida que los datos planteados estén disponibles, bien a través de fuentes secundarias, bien a través de la recolección de datos primarios. En todo caso, consideramos que este trabajo supone un avance en el estudio sistemático de las redes sociales digitales en el sector público, un campo de conocimiento todavía en fase de consolidación dentro de los estudios de administración y gestión pública, pero que cuenta con una creciente conceptualización y resultados de investigación. A continuación, se ofrecen unas conclusiones de carácter preliminar en relación a la propuesta que se ha presentado en este trabajo. 


\section{CONCLUSIONES}

En este artículo se ha realizado una propuesta de modelo para el análisis de las redes sociales digitales en las administraciones públicas. Se ha utilizado como marco de referencia el EEGS de Gil-García (2012), un modelo holístico utilizado para el estudio del gobierno electrónico, pero que también puede ser utilizado para estudiar otras tecnologías de la información y de la comunicación como las redes sociales digitales. No sólo se ha enunciado la propuesta, sino que se ha entrado en el detalle de las diferentes variables necesarias para acometer con éxito un análisis sistemático del éxito en la aplicación de las redes sociales en las organizaciones del sector público.

De la revisión de la bibliografía hemos observado que desarrollando iniciativas para evitar los riesgos descritos por diversos autores, las RSD pueden resultar de utilidad para fortalecer la relación entre los ciudadanos y las instituciones públicas. Su aplicación, podría facilitar alcanzar la fase final de evolución del gobierno electrónico, como sugiere Chun et al. (2010), orientada a la apertura, participación y colaboración. Esta fase final, denominada gobierno 2.0, se caracteriza por la promoción de gobernanza compartida para transformar el modo en el que operan las administraciones públicas, en términos de flujos de información, sin constreñimientos, y en el proceso de toma de decisiones colaborativas usando las tecnologías derivadas de la Web 2.0.

Lo anterior muestra el paso adelante que suponen las redes sociales digitales en la aspiración de lograr unas administraciones públicas más colaborativas, sociales y relacionales, que superen esquemas jerárquicos, burocráticos y eficientistas, En efecto, el potencial de las redes sociales en la gestión pública se concretaría en el aumento del capital relacional de las organizaciones públicas, así como de sus directivos y empleados. Y ello, sin duda, crea valor público, en la medida que incide en la dimensión de legitimidad de la acción pública, no sólo en términos de aproximación a las necesidades reales de los ciudadanos, sino también en la implicación de éstos en los procesos de adopción de decisiones públicas, dentro de lógicas de mayor transparencia, apertura y colaboración.

De manera más específica, el desarrollo de este estudio se enfrenta a diversos desafíos metodológicos que deben ser tenidos en cuenta. En primer lugar, en la propuesta de análisis resultará necesaria la utilización de técnicas estadísticas de análisis, por lo que se hace necesario contar con datos empíricos adecuados y un número de casos suficiente. En segundo lugar, la utilización de Klout Score puede ofrecer algún tipo de sesgo ya que la mayor parte de los indicadores en los que basa su escala no son públicos, si bien, como se ha seńalado previamente, se trata de una 
herramienta ampliamente difundida y utilizada a nivel internacional, que también ha sido empleada en estudios previos. Finalmente, determinadas variables complican el potencial comparado del estudio, en la medida que las organizaciones públicas cuentan con diferencias muy notables entre sí, no sólo en términos de nivel de gobierno, sino también en cuanto a las variedades nacionales.

La propuesta presentada en este artículo se enmarca dentro de un trabajo de investigación doctoral más amplio dirigido al estudio de las redes sociales digitales en las administraciones públicas (en España). La inexistencia de un marco específico para la realización de este estudio, ha derivado en la necesidad de elaborar un esquema analítico que ayude a analizar las redes sociales digitales en las administraciones públicas. Se trata, por lo tanto, de una propuesta inicial que debe ser debatida y mejorada, y cuya validez debe ser contrastada en futuros estudios. En contribuciones posteriores se espera validar la propuesta analítica presentada, mediante la utilización de datos en fase de recopilación por parte de los autores de estas páginas, con la intención ańadida de desarrollar una agenda de investigación en la que se involucre un número cada vez mayor de personas del entorno de habla hispana. 


\section{REFERENCIAS}

Abdelsalam, H. M., Reddick, C. G., Gamal, S. y Al-shaar, A. (2013). Social Media in Egyptian Government Websites: Presence, Usage, and Effectiveness. Government Information Quarterly, 30 (4), 406-416.

Agostino, D. (2013). Using Social Media to Engage Citizens: A Study of Italian Municipalities. Public Relations Review, 39 (3), 232-234.

Anger, I y Kittl, C. (2011) Measuring Influence on Twitter. Ponencia presentada en I-KNOW'11 11th International Conference on Knowledge Management and Knowledge Technologies, Graz, Austria.

Bailey, C. y Singleton, R (2010). National Survey of Social Media Use in State Government. Lexington: NASCIO.

Bannister, F. y Connolly, R. (2012). Forward to the Past: Lessons for the Future of E-Government from the Story so Far. Information Polity, 17 (3-4), 211-226.

Bertot, J. C., Jaeger, P. T., y Grimes, J. M. (2010). Using ICTs to Create a Culture of Transparency: E-Government and Social Media as Openness and Anti-Corruption Tools for Societies. Government Information Quarterly, 27 (3), 264-271.

. (2012a). The Impact of Polices on Government Social Media Usage: Issues, Challenges and Recommendations. Government Information Quarterly, 29 (1), 30-40.

. (2012b). Promoting Transparency and Accountability through ICTs, Social Media, and Collaborative E-Government. Transforming Government People Process and Policy, 6 (1), 78-91.

Bonson, E., Royo, S. y Ratkai, M. (2013). Analysis of European Municipalities' Facebook Channels Activity and Citizens' Engagement. Ponencia presentada en el XVII Congreso AECA, 25-27 de septiembre, Pamplona, España.

Bonson, E., Torres, L., Royo, S. y Flores, F. (2012). Local E-Government 2.0: Social Media and Corporate Transparency in Municipalities. Government Information Quarterly, 29 (2), 123-132.

Boyd, D. M. y Ellison, N. B. (2007). Social Network Sites: Definition, History, and Scholarship. Journal of Computer-Mediated Communication, 13(1), 210-230. 
Chun, S.A., Shulman, S., Sandoval, R. y Hovy, E. (2010). Government 2.0. Making Connections between Citizens, Data and Government. Information Polity. The International Journal of Government \& Democracy in the Information Age, 15 (1-2), 1-9.

Criado, J. I. (2009). Building the Digital Government in Regions: Success Factors and Institutional Barriers in Spanish Comunidades Autónomas. En Cordoba-Pachón, J. R. (Ed.), Systems Thinking and E-Participation: ICT in the Governance of Society. Nueva York: IGI Global.

Criado, J. I., Martín, Y. y Camacho, D. (2011). Experiences Using Social Networks in Spanish Public Administration. Ponencia presentada en 1st International Workshop on Social Data Mining for Human Behaviour Analysis, 26-28 de mayo, Songndal, Noruego.

Criado, J. I., Martínez-Fuentes, G. y Silván, A. (2012) Social media For Political Campaigning. The Use of Twitter by Spanish Mayors in 2011 Local Elections. En Reddick, C. y Aikins, S. (Eds.), Web 2.0 Technologies and Democratic Governance. Nueva York: Springer.

Criado, J. I. y Rojas-Martín, F. (2012). Strategies and Realities of Social Media Diffusion in the Public Sector. Evidence from the Regional Government in Spain. Ponencia presentada en Annual Meeting of the European Group of Public Administration, 5-8 de septiembre, Bergen, Noruega.

Criado, J. I. y Rojas-Martín, F. (Eds.). (2013a). Las Redes Sociales Digitales en la Gestión y las Politicas Públicas. Avances y Desafíos para un Gobierno Abierto. Barcelona: EAPC

Criado, J. I. y Rojas-Martín, F. (2013b). Social Media and Public Administration in Spain. A Comparative Analysis of the Regional Level of Government. En Gil-García, J. R. (Ed.), E-Government Success around the World: Cases, Empirical Studies and Practical Recommendations. Nueva York: IGI Global.

Criado, J. I. y Rojas-Martín, F. (2015). Estrategias y Realidades en la Difusión de las Redes Sociales en las Administraciones Públicas. Análisis desde una Perspectiva Institucionalista. Revista Innovar Journal, 25 (57), 45-62.

De Saulles, M. (2011). Social Media and Local Government in England: Who is Doing That? Ponencia presentada en 11th European Conference on E-Government, 16-17 de junio, Ljubljana, Eslovenia.

Edwards, C., Spence, P.R., Gentile, C.J. y Edwards, A. (2013). How Much Klout Do You Have ... A Test of System Generated Cues on Source 
Credibility. Computer in Human Behaviour, 29 (5), A12-A16.

Ellison, N. y Hardey, M. (2014). Social Media and Local Government: Citizenship, Consumption and Democracy. Local Government Studies, 40(1), 21-40.

Fountain, J. E. (2001). Building the Virtual State: Information Technology and Institutional Change. Washington, D.C.: Brooking Institution Press.

Gascó, M. (2009). El Papel de las Instituciones en el Desarrollo del Gobierno Electrónico en América Latina: Algunas Reflexiones. Estado, Gobierno, Gestión Pública. Revista Chilena de Administración Pública, 14, 37-59.

Gascó, M., Tamyko Y., Longo, F. y Fernández, C (2012). La Gestió de la Interoperabilitat en Govern Electrònic a les Administracions Publiques Catalanes. Barcelona: Institut de Governança i Direcció Pública.

Gil-García, J. R. (2012). Enacting Electronic Government Success: An Integrative Study of Government-Wide Websites, Organizational Capabilities, and Institutions. Nueva York: Springer.

Graham, M. y Avery, E. (2013). Government Public Relations and Social Media: An Analysis of the Perceptions and Trends of Social Media Use at the Local Government Level. Public Relations Journal, 7 (4), 1-21.

Grimmelikhuijsen, S. (2012). A Good Man but a Bad Wizard. About the Limits and Future of Transparency of Democratic Governments. Information Polity 17 (3-4), 293-302.

Heidinger, C., Buchmann, E. y Klemens, B. (2010). Impact Assessment in Public Policy: Towards a Web 2.0 Application. Information Polity, $15(1,2), 33-50$.

Hofmann, S., Beverungen, D., Räckers, M. y Becker, J. (2013). What Makes Local Governments' Online Communications Successful? Insights from a Multi-Method Analysis of Facebook. Government Information Quarterly, 30 (4), 387-396.

Kaplan, A. M. y Haenlein, M. (2010). Users of The World, Unite! The Challenges and Opportunities of Social Media. Business Horizons, 53 (1), 59-68.

Klout (2015). Sobre Klout. Disponible en https://klout.com/corp/about [15-06-2015] 
Lin Y. R., Bagrow, J.P. and Lazer, D. (2011). More Voices Than Ever? Quantifying Media Bias in Networks. Ponencia presentada en Conference on Weblogs and Social Media, P.o.t.F.I.A.C.o.W.a.S., 17-21 de julio, Barcelona, España.

Linders, D. (2012) : From E-Government to We-Government : Defining a Typology for Citizen Coproduction in the Age of Social Media. Govnerment Information Quarterly, 29 (4), 446-454.

Lips, M. (2012). E-Government is Dead : Long Live Public Administration 2.0. Information Polity, 17 (3-4), 239-250.

Luna-Reyes, L. F. (2006). E-Government Measurement and Evaluation. 7th International Conference on Digital Government Research, 21-24 de mayo, San Diego, California, Estados Unidos.

Luna-Reyes, L. F. y Gil-Garcia, J. R. (2011). Using Institutional Theory and Dynamic Simulation to Understand Complex E-Government Phenomena. Government Information Quarterly, 28 (3), 329-345.

Luna-Reyes, L. F., Mellouli, S. y Bertot, J. C. (2013). Key Factors and Processes for Digital government success. Information Polity, 18 (2), 101-105.

Magnusson, M., Bellström, P. y Thoren, C. (2012). Facebook Usage in Government - A Case Study of Information Content. Ponencia presentada en 18th Americas Conference on Information Systems (AMCIS 2012), 9-12 de Agosto, Seattle, Washington, Estados Unidos.

Mainka, A., Hartmann, S., Stock, W. G. y Peters, I. (2014). Government and Social Media: A Case Study of 31 Informational World Cities. Ponencia presentada en 47th Hawaii International Conference on System Sciences, 6-9 de enero, Waikoloa, Hawaii, Estados Unidos.

Maultasch Oliveira, G. H. y Welch, E. W. (2013). Social Media Use in Local Government: Linkage of Technology, Task, and Organizational Context. Government Information Quarterly, 30 (4), 397-405.

Meijer, A. y Thaens, M.. (2013). Social Media Strategies: Understanding the Differences Between North American Police Departments. Government Information Quarterly. 30 (4), 343-350.

Mergel, I. (2012a). Social Media in the Public Sector: A Guide to Participation, Collaboration, and Transparency in the Networked world. San Francisco: John Wiley \& Sons. 
.(2012b). The Social Media Innovation Challenge in the Public Sector. Information Polity, 17 (3-4), 281-292.

(2013). A Framework for Interpreting Social Media Interactions in the Public Sector. Government Information Quarterly, 30 (4), 327-334.

Mickoleit, A. (2014). Social Media Use by Governments: A Policy Primer to Discuss Trends, Identify Policy Opportunities and Guide Decision Makers. OECD Working Papers on Public Governance, 26, OECD.

Mossberger, K., Wu y. y Crawford, J. (2013)- Connecting Citizens and Local Governments? Social Media and Interactivity in Major U.S. Cities. Government Information Quarterly, 30 (4), 351-358.

Nam, T. (2012). Suggesting Frameworks of Citizen-Sourcing via Government 2.0. Government Information Quarterly, 29 (1), 12-20.

Orlikowski, W. y Iacono, C. S. (2001). Research commentary: Desperately Seeking the "IT" in IT Research-A call to Theorizing the IT Artifact. Information Systems Research, 12 (2), 121-134.

Osimo, D (2008) Web 2.0 in Government: Why and How? JRC Scientific and Technical Reports. Seville: European Commission.

Panagiotopoulos, Panagiotis Sams, S. (2012). An Overview Study of Twitter in the UK Local Government. Ponencia presentada en TGov 2012, 8-9 de mayo, Brunel University, Uxbridge, Reino Unido.

Picazo-Vela, S., Gutiérrez-Martínez, I. y Luna-Reyes, L. F. (2012). Understanding Risks, Benefits, and Strategic Alternatives of Social Media Applications in the Public Sector. Government Information Quarterly, 29 (4), 504-511.

Song, C. y Lee, J. (2013). Can Social Media Restore Citizen Trust in Government? Ponencia presentada en 11th Public Management Resaerch Conference, 20-22 de junio, Wisconsin, Madison, Estados Unidos.

Welch, E. y Feeney, M. (2013). Technology-Task Coupling: How Social Media Use is Related to Public Managers' Perceptions of E-Government Outcomes. Ponencia presentada en 11th Public Management Resaerch Conference, 20-22 de junio, Wisconsin, Madison, Estados Unidos.

Recibido: 15-05-2015

Aceptación de la versión final: 21-06-2015 